\title{
Upgrade of the ATLAS Level-1 Calorimeter Trigger
}

\author{
Martin Wessels on behalf of the ATLAS Collaboration
}

\begin{abstract}
The Level-1 Calorimeter Trigger (L1Calo) of the ATLAS experiment has been operating well since the start of LHC data taking, and played a major role in the Higgs boson discovery. To face the new challenges posed by the upcoming increases of the LHC proton beam energy and luminosity, a series of upgrades is planned for L1Calo. The initial upgrade phase in 2013-14 includes substantial improvements to the analogue and digital signal processing to allow more sophisticated digital filters for energy and timing measurement, as well as compensate for pile-up and baseline shifting effects. Two existing digital algorithm processor subsystems will receive substantial hardware and firmware upgrades to increase the real-time data path bandwidth, allowing topological information to be transmitted and processed at Level-1. An entirely new subsystem, the Level1 Topological Processor, will receive real-time data from both the upgraded L1Calo and Level-1 Muon Trigger to perform trigger algorithms based on entire event topologies. During the second upgrade phase in 2018-19, major parts of L1Calo will be rebuilt in order to exploit a tenfold increase in the available calorimeter data granularity compared to that of the current system. The calorimeter signals will be received via optical fibres and distributed to two distinct processing systems. Those systems apply sliding window algorithms and quasi-offline algorithms to achieve object reconstruction and identification. The algorithms are implemented on high-density electronics boards which make use of recent developments in high speed data transmission and FPGA technology. The expected performance improvements are presented together with the upgraded hardware and firmware implementations. The status of the prototypes, integration and commissioning efforts are also reviewed.
\end{abstract}

\section{INTRODUCTION}

A $\mathrm{T}$ the end of 2012, the first $p p$ run of the LHC ended successfully with a centre-of-mass energy of $8 \mathrm{TeV}$ and peak luminosities routinely up to $0.77 \times 10^{34} \mathrm{~cm}^{-2} \mathrm{~s}^{-1}$. More than $20 \mathrm{fb}^{-1}$ of data were collected by each the ATLAS and CMS experiments, resulting in the discovery of the long sought-after Higgs boson in mid-2012. When the LHC resumes operation in 2019 after the second long shutdown, it will produce collisions at a centre-of-mass energy at or near its ultimate design of $14 \mathrm{TeV}$. The instantaneous luminosity will increase beyond $10^{34} \mathrm{~cm}^{-2} \mathrm{~s}^{-1}$ and the associated pileup will also increase. This offers a major gain in physics potential through a substantial improvement in mass reach, with a dataset after three years of running totalling $300 \mathrm{fb}^{-1}$, more than ten times that which has been recorded to date.

The ATLAS detector has to be upgraded to exploit the new physics reach, preserve the physics acceptance, and to meet the challenges of the high-luminosity environment. The upgrades

Manuscript received May 22, 2014. This research work was supported by the Bundesministerium für Bildung und Forschung (BMBF FZK: 05H12VH2) and the Helmholtz Alliance Physics at the Terascale.

Martin Wessels is with the Kirchhoff Institute für Physik, RuprechtKarls-Universität Heidelberg, D-69120 Heidelberg, Germany (e-mail: martin.wessels@cern.ch). that are being prepared for ATLAS operations after 2018 are referred to as Phase-I upgrades. The terms LS1, LS2 refer to the two long shutdowns of the LHC in 2013-14 and anticipated in 2018, during which the detector upgrades occur ${ }^{1}$.

This note describes the Phase-I upgrades of the ATLAS Level-1 Calorimeter Trigger [1].

\section{The ATLAS LeVEl-1 CAlorimeter Trigger}

$\eta$ HE Level-1 Calorimeter Trigger [2] (L1Calo) is a pipelined system processing signals from the ATLAS experiment's main electromagnetic and hadronic calorimeters in real time to produce trigger signals to the Central Trigger Processor (CTP). It consists of three main sub-systems: the PreProcessor, the Cluster Processor and the Jet/Energy Processor.

The 7168 analogue input signals are first conditioned, digitised and associated to a particular LHC bunch crossing in the L1Calo PreProcessor system [3]. Digital transverse energies for every so-called trigger tower are then transmitted in parallel to the two separate processor systems which run the trigger algorithms [4], likewise in parallel. The Cluster Processor (CP) uses the full trigger tower granularity in the central region to search for small localised clusters typical of electron, photon and tau candidates. The Jet/Energy Processor (JEP) operates on so-called jet elements with a granularity of $2 \times 2$ sums of electromagnetic and hadronic trigger towers to identify jet candidates and to form global transverse energy sums: missing, total and jet-sum transverse energy and missing transverse energy significance.

For both the $\mathrm{CP}$ and the JEP systems, the results from entire crates of modules are merged using common merger modules (CMM) providing system-wide counts of objects and sums which are compared to programmable thresholds. During Run $1^{2}$, up to a maximum of 116 result bits were sent to the CTP where the Level-1 (L1) trigger decision is formed. For all events that are selected by L1, a programmable selection of data from L1Calo is read out via Readout Driver modules to the ATLAS data acquisition system. On request, data are also sent to the Level-2 Trigger RoI Builder (RoIB) for further use by the HLT trigger algorithms.

In order to manage the increasing rates after the upgrade of the LHC, whilst preserving an effective and efficient calorimeter trigger, significant upgrades will be necessary.

\section{A. Architecture Evolution}

Figure 1 gives an overview of the L1 trigger system for Run 3, with L1Calo set in the context of other system

\footnotetext{
${ }^{1}$ Another Phase-II upgrade of the LHC machine and detectors will take place during the long shutdown LS3 which is anticipated in 2022.

${ }^{2}$ Run 1, Run 2, Run 3 refer to the periods of data-taking operation of ATLAS before and between the long shutdowns, LS1, LS2 and LS3.
} 


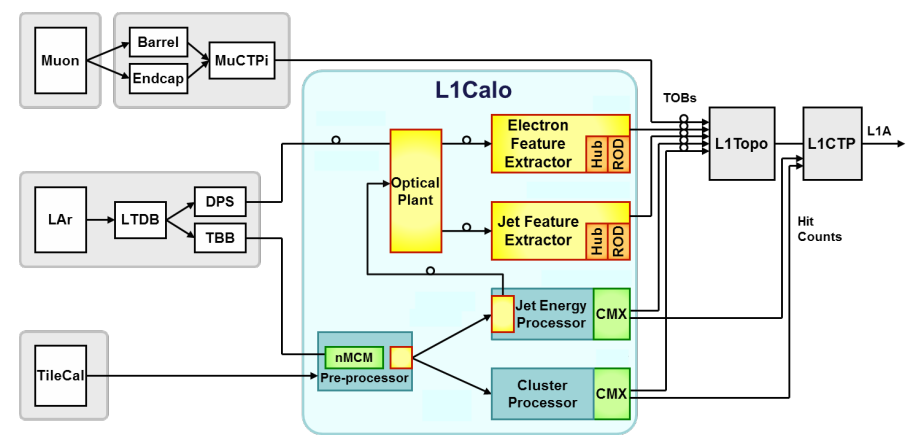

Fig. 1. Overview of the Level-1 trigger system planned for Run 3.

components. New signal digitisation in the current PreProcessor system will improve bunch crossing identification and $E_{T}$ estimation in the face of increased pile-up and will be based on a replacement of the Multi-Chip Modules (MCM). The existing CMMs in the CP and JEP systems will be replaced with new Common Merger Modules (CMX) to enable transmission of position and transverse energy estimates to a new Topological Processor (L1Topo), in addition to existing count-above-threshold information sent to the CTP. L1Topo will run algorithms to flag interesting event topologies to the CTP. Algorithms will rely on event features such as opening angles between Trigger Objects (TOBs), effective masses of TOB pairs, etc. These upgrades will be installed during LS1 to meet anticipated triggering challenges in the subsequent run.

For Run 3 the CP and JEP systems will be replaced with new hardware and algorithms working with finer granularity information. Liquid Argon Calorimeter (LAr) signals will be digitised by a new Digital Processing System (DPS) to produce $E_{T}$ measurements at a finer granularity [5]. Information from the Tile Calorimeter will be derived from the existing digitisation system (within L1Calo) at the maximum granularity available there. These data will be sent to the electromagnetic and jet feature extractor subsystems, eFEX and jFEX respectively, over multi-gigabit optical fibre links. The eFEX subsystem will employ new cluster-finding algorithms on the higher granularity data to produce more refined TOBs. The jFEX subsystem will use jet-finding algorithms on higher granularity data and summing over a wider region.

\section{B. Input Data}

The $0.1 \times 0.1$ trigger towers ${ }^{3}$, as used in the present $\mathrm{L} 1$ Calorimeter Trigger and formed by analogue summation of calorimeter cells, are too coarse to handle the increased levels of pile-up expected for the upgraded LHC. The primary aim of the LAr electronics upgrade is to make finer-granularity data available to the trigger electronics.

In the proposed design, the DPS will provide information from up to ten so-called SuperCells within each electromagnetic trigger tower, where a SuperCell is a sum of four or eight calorimeter cells. As shown in Figure 2, each $0.1 \times 0.1$ tower will contain one SuperCell from layers zero (Pre-Sampler)

\footnotetext{
${ }^{3}$ Regions in the detector are given in units of pseudo-rapidity $\eta$ and azimuth $\phi$, and are specified as $\Delta \eta \times \Delta \phi$
}

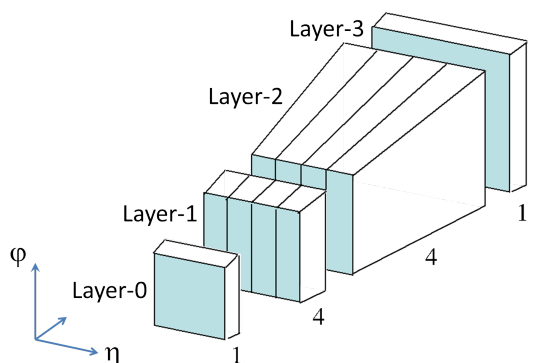

Fig. 2. The trigger granularity from each $0.1 \times 0.1$ trigger tower after the upgrade of the LAr Calorimeter electronics. Ten $E_{T}$ values are provided from "1-4-4-1" longitudinal/transverse samples, each forming a SuperCell.

and three (back sample) of the EM calorimeter, and four SuperCells of finer granularity in layers one and two.

\section{RUn 2 System ARChitecture}

A LREADY during LS1, a number of upgrades will be made to L1Calo. In the PreProcessor system the MCMs, which digitise, calibrate and filter the calorimeter signals, will be replaced with newer modules that provide $80 \mathrm{MHz}$ digitisation, lower noise and greater flexibility to handle pileup. Downstream of this, L1Calo will be enhanced to allow topological triggers; the firmware on the CPMs and JEMs will be modified and the merger modules will be replaced. This is shown in Figure 3.

\section{A. The New Multi-Chip Module}

Most of the signal processing in the initial PreProcessor subsystem is performed in MCMs. Each MCM receives and digitises four analogue calorimeter trigger signals, extracts the $E_{T}$ for each signal, assigns the correct bunch crossing, and sends the results serially to the CP and JEP subsystems.

During LS1, the MCM will be replaced with a new device, the new MCM (nMCM). In this plug-compatible device, the ASIC is replaced by an FPGA. The four inputs from the motherboard are digitised to ten-bit precision at $80 \mathrm{MHz}$ in two dual-channel ADCs. In general the signal processing runs at the LHC clock frequency of $40 \mathrm{MHz}$, discarding every other 10-bit data word. However, saturated-pulse processing uses the full $80 \mathrm{MHz}$ samples.

The results of the digital signal processing are transmitted serially on LVDS links to the CP and JEP subsystems at 480 Mbaud. Data is sent to the $\mathrm{CP}$ for individual $0.1 \times 0.1$ trigger towers, while the JEP is sent data pre-summed into $0.2 \times 0.2$ jet elements. For Run 3 operation, the serial link to the JEP system will be upgraded to 960 Mbaud, to carry $0.1 \times 0.1$ hadronic $E_{T}$ values for onward transmission to eFEXs and jFEXs. The nMCM also includes a programmable analogue signal generator which may be used for standalone tests.

Use of an FPGA gives flexibility to the system, for example in the choice and sign of digital filter coefficients, and the possibility of using different types of filter. Also, in the Run 1 PreProcessor, the same $E_{T}$ values are sent to the CP and JEP subsystems, with a single look-up table used for calibration. Including a second look-up table would allow provision of 


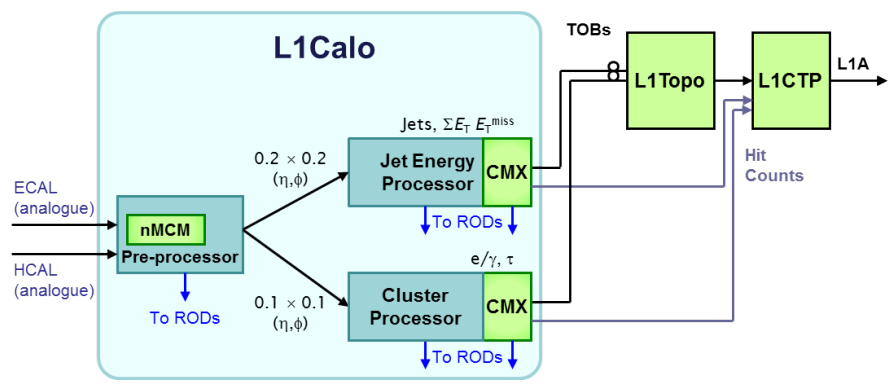

Fig. 3. System Architecture during Run 2. New components are shown in green

separate, hadronically calibrated $E_{T}$ values to the JEP. The saturated BCID algorithm can be improved using the $80 \mathrm{MHz}$ samples from the nMCM, to ensure the correct BCID up to the highest possible energies. Finally, the nMCM offers the possibility to correct for pile-up induced fluctuations of the pedestal. Such a correction would greatly increase the performance of all triggers which are affected by pile-up, particularly $E_{T}^{\text {miss }}$ triggers.

\section{B. The Extended Common Merger Module}

The extended Common Merger module (CMX) is a replacement for the CMM and supports all Run 1 CMM functionality. This is enhanced in two important ways, with faster communication over existing data paths and provision of inputs to L1Topo.

The CMX provides all the multiplicity-based L1Calo trigger algorithms by counting the number of TOBs above threshold. Input data from the $\mathrm{CP}$ modules (CPMs) and JEP modules (JEMs) arrive at $160 \mathrm{Mb} / \mathrm{s}$ (instead of $40 \mathrm{Mb} / \mathrm{s}$ ), sufficient bandwidth to permit TOB information to be sent in replacement of counts over threshold. Threshold-dependent isolation criteria are applied to data from the CPMs. At each jet threshold, $E_{T}$ calculations of two different jet sizes are performed on data from the JEMs. $E_{T}$ thresholds are defined for particular TOB types, along with other criteria which may be applied to the TOBs. Firmware upgrades in the CPMs and JEMs are necessary to support these changes.

The total multiplicity for each TOB type and threshold is calculated by producing crate-level counts, in crate CMXs. These are sent over LVDS links to one of the CMXs to form a system-wide sum which is then passed to the CTP. The large logic capacity of the CMX FPGAs permits approximately twice as many selection and isolation thresholds for electromagnetic and tau clusters and similarly doubles the number of jet thresholds when compared to the CMM.

In addition to the multiplicity analysis of TOBs, the CMX also collects and manipulates the TOB information and sends it on $6.4 \mathrm{~Gb} / \mathrm{s}$ fibres to the L1Topo processor. Zero suppression of candidates will be performed in each CMX in parallel, before grouping the candidates by output fibre. This simplifies the first stages of input handling in L1Topo.

ROI and DAQ read-out of the CMX is supported over fibres running the G-link protocol and using existing RODs. Board timing, monitoring and initialisation are supported in a similar manner to the CMM.

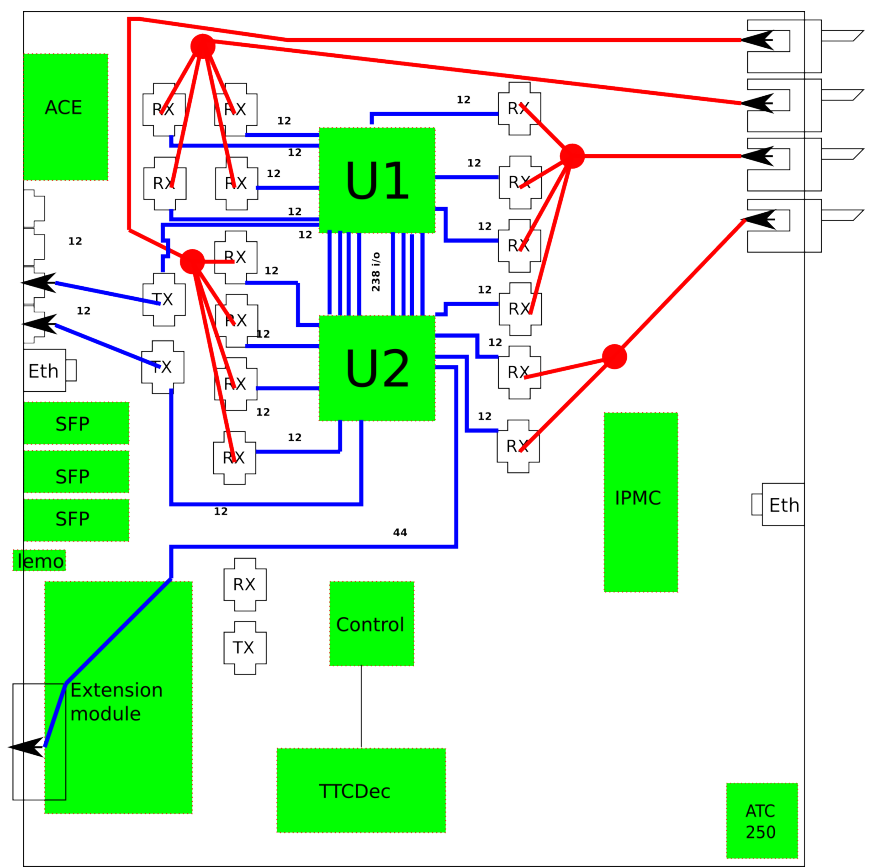

Fig. 4. Layout of the L1Topo module, showing the main functional blocks and real-time data path lines.

\section{The Level-1 Topological Trigger}

The Topological Processor for the L1 trigger performs realtime event selection based on the geometrical relationship between trigger objects such as muons, jets, $E_{T}^{\text {miss }}$, electron and tau clusters, and selections based on the variables $H_{T}$, $M_{\text {eff }}$ or $M_{\text {inv }}$.

The baseline implementation of the L1Topo system is a single ATCA shelf equipped with two or more L1Topo processor modules. They receive data from the L1 Muon and Calorimeter trigger systems via optical fibres. The real-time output from L1Topo is sent to the CTP. Figure 4 shows the layout of the L1Topo processor module. Event selection is performed by two FPGAs (labelled U1 and U2 in the figure), receiving their input via optical receivers. Module control and read-out functionality are implemented in the separate Control FPGA.

The input to a L1Topo module is via four 48-way fibre ribbon bundles into four MTP-CPI connectors. From these connectors, the optical signals are routed via octopus cable sets to the twelve Avago MiniPOD optical receivers (labelled $\mathrm{Rx}$ in the figure) that perform opto-electrical conversion. The electrical signals are routed into the processing FPGAs via their on-chip multi-gigabit transceivers (MGT) and deserialised at the LHC clock frequency. Using the baseline link speed of $6.4 \mathrm{~Gb} / \mathrm{s}$ with standard $8 \mathrm{~b} / 10 \mathrm{~b}$ encoding provides $\sim 5.1 \mathrm{~Gb} / \mathrm{s}$ of payload for each of the 160 inputs to the two main FPGAs. It should be noted that both the MGTs and optical receivers can operate at up to $13 \mathrm{~Gb} / \mathrm{s}$.

Many topological trigger algorithms are under study. It is not yet clear which algorithms will be chosen, and the selection may change with luminosity. The firmware is therefore developed in a modular structure, isolating the algorithmic core from the handling of FPGA I/O, and providing generic 


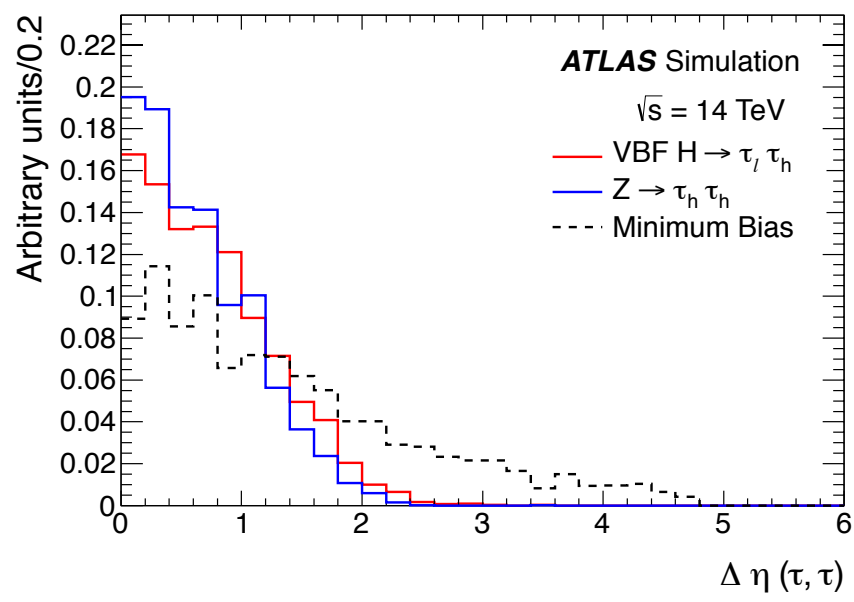

Fig. 5. The distribution of $\Delta \eta$ for tau candidates from the decay $H \rightarrow \tau \tau$ for $m_{H}=125 \mathrm{GeV}$ Higgs produced in the VBF process; the leading tau exceeding an online threshold of $20 \mathrm{GeV}$ and the sub-leading tau exceeding an online threshold of $12 \mathrm{GeV}$.

functions such as sorting of input trigger objects. The output consists of individual bits indicating the results of each specific topological trigger algorithm.

In Run 2, the read-out to the ROI builder and DAQ is implemented in the control FPGA. The Read-Out Links are based on the 2Gb/s HOLA S-LINK protocol for compatibility with the existing system. An additional read-out path is provided directly from the processor FPGAs onto the backplane. This link is compatible with the L1Calo Hub module which will form part of the Run3 upgrade architecture, and provides access to higher read-out speeds and additional readout processing needed after LS3.

Before and after the Phase-I upgrade, the largest impact of the topological trigger will be on di-tau final states (primarily for Higgs studies) with one or both taus decaying hadronically. The rate of most di-tau triggers can be reduced to a tolerable level by imposition of $\Delta \eta$ or $\Delta \phi$ and $\Delta R$ cuts with modest loss in efficiency for signal events. An approach, unbiased with respect to production mechanism, is to apply the $\Delta \eta$ cut to the two $\tau$ objects. With the topological selection of $\Delta \eta<2.0$, the rate is reduced by $25 \%$ without losing almost any signal events, as shown in Figure 5.

\section{Run 3 System ARChitecture}

D URING LS2 there will be further upgrades, both to L1Calo and the upstream calorimeter electronics. Two new subsystems will be added to L1Calo: the electromagnetic and jet feature extractors, see Figure 6. To achieve the increased discriminatory power necessary to handle the LHC luminosities planned beyond LS2, these will process calorimeter data at a finer granularity than the pre-LS2 L1Calo system.

Prior to LS2, the LAr and Tile calorimeter electronics provide analogue data which are digitised in the PPMs. During Run 3 the digitised LAr calorimeter data are provided to L1Calo by the DPS. Analogue Tile calorimeter data are digitised in an upgraded PPM, using the nMCM fitted in LS1.

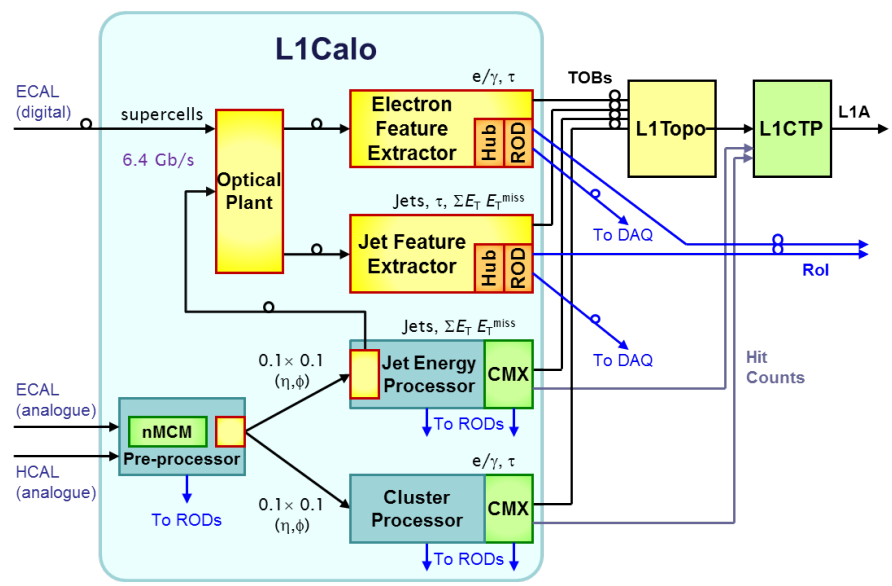

Fig. 6. System Architecture during Run 3. New components are shown in yellow / orange.

\section{A. Input Interfaces}

Both the eFEX and jFEX systems receive digitised $E_{T}$ values on optical fibres. The EM, hadronic endcap (HEC) and forward calorimeter (FCAL) inputs are all provided by the LAr DPS. However, the TileCal will continue to provide analogue trigger signals, which are digitised in the PPMs and transmitted to the JEMs by new PPM LVDS Cable Driver (LCD) daughter cards. JEM input daughter cards will be built to provide optical signals to the FEXs, necessitating a corresponding increase in the PPM to JEM link transmission speed.

In the baseline design, the inputs to the eFEX and jFEX systems are assumed to be $6.4 \mathrm{~Gb} / \mathrm{s}$ links. Higher link speeds, such as $10 \mathrm{~Gb} / \mathrm{s}$, are under investigation and will be used if tests prove successful. If this were possible the additional bandwidth might be used to send data for a larger number of towers per fibre, to increase the dynamic range of transmitted data, or to reduce the need for data compression.

Each FEX module processes a fixed core area in $\eta-\phi$. To do this, it also needs copies of data for the surrounding SuperCells or towers required by the sliding window algorithms. Electrical re-transmission of multi-gigabit signals between modules over the backplane is precluded by latency and board layout considerations, so the environment data need to be duplicated at source and sent to each module. This will mainly be done by driving two separate copies of the same data on separate fibres from source FPGAs. Since the organisation of fibres in ribbons from the DPS will in general be different from that needed by each eFEX or jFEX module, an intermediate optical patch panel is needed between the DPS outputs and the FEX inputs.

\section{B. The Electron Feature Extractor Module}

The function of the eFEX module is to identify isolated energy deposits indicative of electrons, photons and tau particles. As shown in Figure 7, this is done in a bank of four Processor FPGAs, and the results transmitted optically to L1Topo. On receipt of an L1A, data are copied from the real-time path to the read-out path and from there to a ROD daughter card 


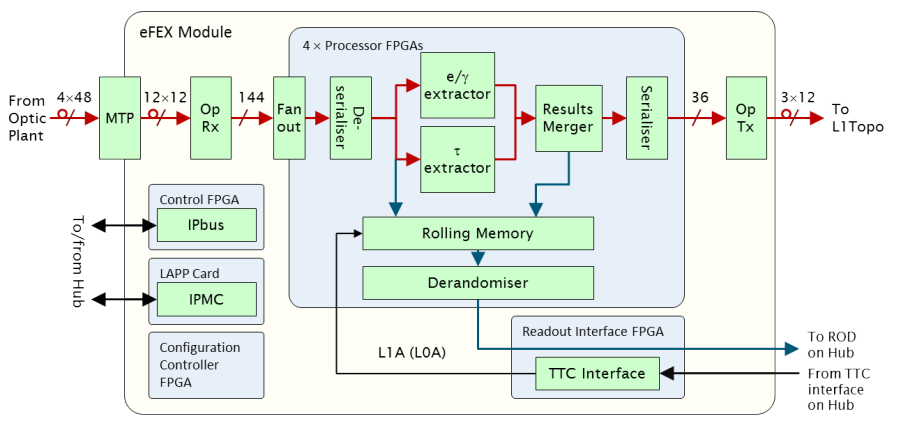

Fig. 7. Block diagram of the eFEX module showing the real-time and readout data paths. Control and monitoring signals are omitted except for the L1A.

mounted on a Hub module. The eFEX is an ATCA module, conforming to the PICMG 3.0 Revision 3.0 specification.

The algorithms used by the eFEX define a core area in which energy deposits are found, surrounded by an environment area used to establish isolation. Each eFEX processes a core area of up to $1.7 \times 0.8$ of calorimeter data, for which it receives environment data covering $1.8 \times 1.0$. A total of 24 eFEX modules are required to process all of the data from the calorimeter within the range $\eta<|2.5|$.

To process an environment of $1.8 \times 1.0$, each eFEX module must receive electromagnetic calorimeter data on at least 90 fibres and hadronic calorimeter data on at least 25 fibres. The baseline design uses 100 and 36 fibres respectively to allow for the partial use of the data on fibres at module boundaries. A total of 136 multi-Gb/s signals are received from the calorimeters (excluding spares). Of these, 52 are transmitted from the MiniPODs to a single Processor FPGA, 72 are transmitted to two Processor FPGAs (required by the overlapping nature of the eFEX algorithms) and 12 are transmitted to three FPGAs. The eFEX must therefore handle upwards of $230 \mathrm{multi}-\mathrm{Gb} / \mathrm{s}$ signals.

Most of the eFEX functionality, including all the real-time functionality, is implemented in the four Xilinx Processor FPGAs. Each one processes a $0.6 \times 1.0$ region, receiving calorimeter data on 58 serial inputs at $6.4 \mathrm{~Gb} / \mathrm{s}$ and identifying $e / \gamma$ and $\tau$ objects using sliding window algorithms. For each object found, a TOB datum is generated, comprising the object type, the energy measured and the $\eta$ or $\phi$ coordinates. The FPGA also records the input and output data in scrolling memories and, on receipt of a L1A signal, transfers the data for the triggered bunch crossing to a derandomising buffer. Here a data packet is built and transmitted it to the Read-out Interface FPGA.

In the Run 3 algorithms, electromagnetic clusters are built around a seed - a SuperCell in layer two of the electromagnetic calorimeter, where most of the shower energy is deposited and which is more energetic than any of the surrounding SuperCells. A cluster is formed by summing this cell with the more energetic of its neighbours in the $\phi$ direction, then summing both neighbours in the $\eta$ direction, to form a cluster of $3 \times 2$ SuperCells. The corresponding cells from layer one are added, plus the pair of cells in layers zero and three corresponding to the towers containing the seed and its

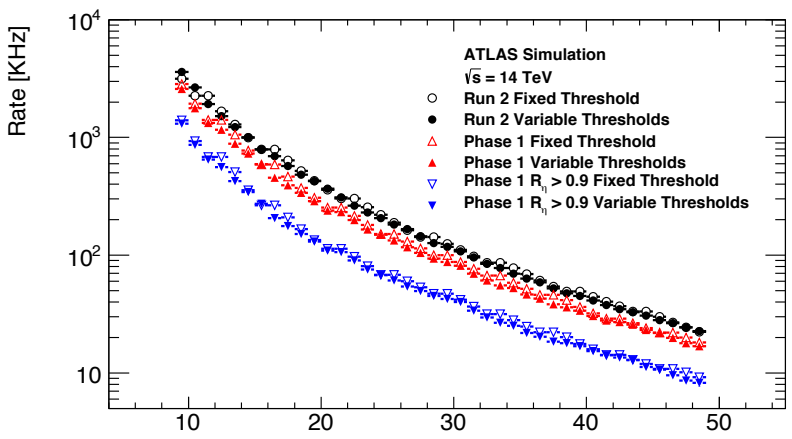

Offline electron pT for which this set of cluster cuts is $95 \%$ efficient [GeV]

Fig. 8. EM trigger rates for Run 2 and Run 3 systems. The $E_{T}$ scale is the offline electron $p_{T}$ for which the $E_{T}$ thresholds used would give $95 \%$ efficiency.

neighbour in $\phi$.

Figure 8, shows the rate vs $E_{T}$ for the EM cluster alone in Run 3, compared to the Run 1 trigger algorithm, in simulated Run 3 conditions. Without any additional vetoes, the proposed Run 3 system produces a $\sim 20 \%$ lower rate for the same efficiency.

The greatest gain in jet rejection comes from use of the finer granularity of the EM calorimeters in layers 1 and 2 to build more powerful jet vetoes. The example that has proved most successful is an " $R_{\text {core" }}$ " variable, defined as the ratio of the $E_{T}$ in an inner cluster to the total in a larger region which includes the core. A narrow EM object should be mostly contained within the inner cluster, resulting in higher values of $R_{\text {core }}$ than are typically produced by more extended QCD jets. Figure 9 shows $R_{\text {core }}$ distributions for electrons from $Z \rightarrow e^{+} e^{-}$events and for jets passing a cluster $E_{T}$ threshold of $20 \mathrm{GeV}$. Here, an inner core of $3 \times 2$ SuperCells is compared to a larger environment of $7 \times 3$ SuperCells. Another specific example of this is the $R_{\eta}$ variable as used in Figure 8, which in the Run 3 trigger is defined as the ratio of $3 \times 2$ SuperCells to a $7 \times 2$ SuperCell window.

\section{The Jet Feature Extractor Module}

The jFEX processor system performs jet, large-area tau, $E_{T}^{\text {miss }}$ and total- $E_{T}$ trigger algorithms on $0.1 \times 0.1$ electromagnetic and hadronic towers. The jFEX system consists of a single ATCA shelf, equipped with several module types and fed by the optical fibre plant. The fibres are routed via rear transition modules into jFEX modules, where the physics algorithms are run. Timing distribution, read-out and control functionality are implemented on two Hub modules located in the fabric slots of the shelf.

In the baseline design, each jFEX module processes calorimeter data from a $\phi$-octant, covering the entire $\eta$ range, including barrel, endcap and forward calorimeters. A total of eight jFEX modules is required for full coverage of the calorimeter solid angle. Jets and taus are identified on the jFEX modules as local maxima of energy deposits in a sliding window of $0.9 \times 0.9$. This requires that each $\mathrm{jFEX}$ module is supplied with a copy of the data from neighbouring $\phi$-octants. The data duplication is performed at the data source, the DPS. 


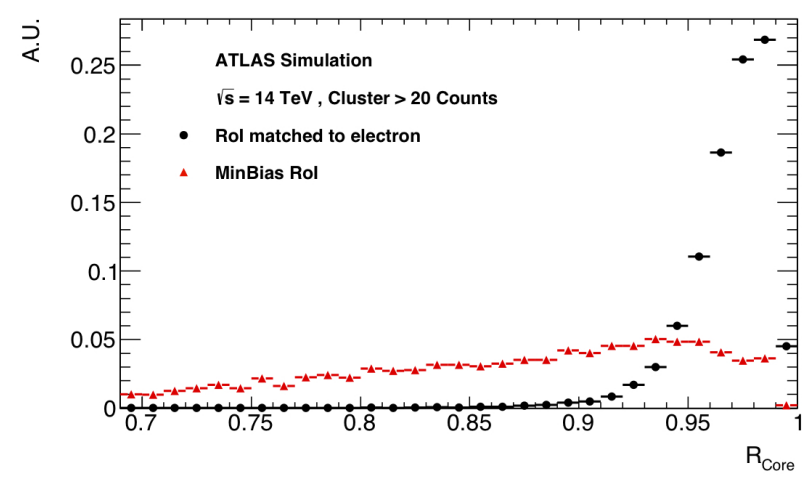

Fig. 9. $R_{\text {core }}$ distributions for clusters matched to reconstructed electrons and for clusters found in minimum-bias QCD events.

There is a strong interest in considerably higher input data rates $(9.6 \mathrm{~Gb} / \mathrm{s}$ or above) compared to the baseline design, which would support significantly larger jet sizes. Initial studies indicate that the $\mathrm{jFEX}$ system could utilise an increased transmission speed to reach a jet size of $1.7 \times 1.7$, corresponding to a jet radius parameter of 0.85 . Furthermore, a remapping of the calorimeter signals would provide each jFEX module with a slice in $\eta$ (full $\phi$ coverage), allowing each jFEX to perform event-by-event pile-up subtraction in slices of $\eta$ to improve $E_{T}^{\text {miss }}$ determination, and to correct the jet energies measured within the jFEX system.

A diagram of the jFEX module is shown in Figure 10. The design foresees a flexible definition of jet algorithms, allowing for several definitions to be used concurrently. The information transmitted from the jFEX system to L1Topo allows areabased pile-up correction of the jet energies.

Real-time input data from the DPS (including LAr EM, HEC \& FCAL) and input from the JEM daughter board (TileCal) enter the jFEX module, via the rear transition module. This provides the mechanics for feeding fibres into the jFEX module on four 72-way MTP/MPO connectors in ATCA zone 3 . This allows a total of 288 input fibres per jFEX. On the jFEX module, the four fibre bundles are each split into six twelve-way ribbons that are received in MicroPOD electrooptical devices. These devices support data rates greater than $10 \mathrm{~Gb} / \mathrm{s}$ per fibre. The MicroPOD outputs are routed, as differential signals, to the GTH high-speed receivers in the Processor FPGAs.

The Processor FPGAs identify and report TOBs (i.e. jet and large-area tau candidates) and calculate global event quantities (e.g. $\left.E_{T}^{\mathrm{miss}}\right)$. This is done separately in segments of $\eta$, so as to allow for downstream pile-up corrections. All six Processor FPGAs perform these calculations. In addition one Processor FPGA consolidates the results before they are sent, optically via MicroPOD devices, to L1Topo.

In the jFEX the finer granularity of the SuperCells is not available. However, compared with the current system, there is overall still an improvement in spatial granularity and also in least count, with a digit scale of $250 \mathrm{MeV} /$ count rather than $1 \mathrm{GeV}$, and more importantly an improved ability to resolve small signals from noise.

The nature of the L1 trigger means that offline-style jet-

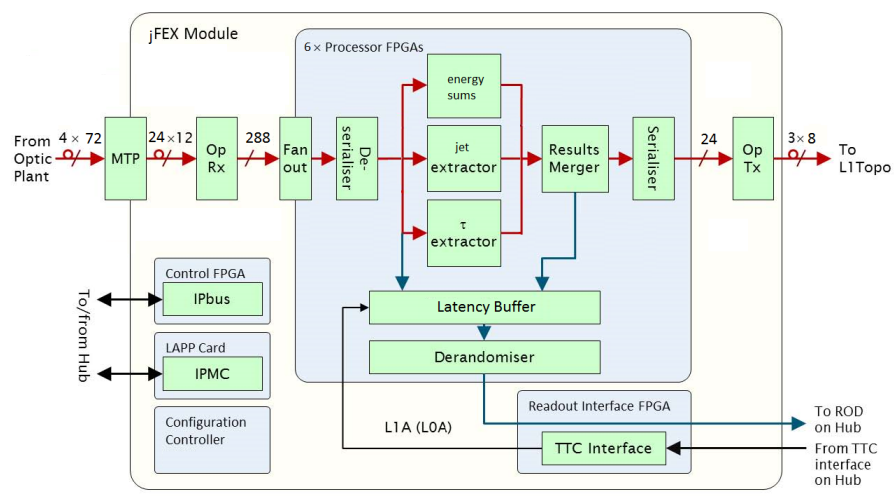

Fig. 10. Block diagram of the jFEX module.

finders, which sequentially search for jets, removing elements used in each jet before searching for the next, are not suitable. At L1, which must identify all possible jets in a limited and fixed latency, jet algorithms must be executed in parallel within a set of overlapping window environments, and cannot use information external to those environments. The size of the environment is limited by the available bandwidth and the necessary interconnectivity to share data between FPGAs, modules and crates. A $9 \times 9$ tower environment, as proposed in the baseline design, has an area slightly larger than a $\Delta R=0.4$ cone. Since the windows overlap, it is necessary to identify which windows should be considered as jet candidates.

A technique that has been used in the current trigger is to seed the jet using a smaller cluster, which can be identified as a maximum using the information in the window, and then to build a larger cluster around this to estimate the jet $E_{T}$. One simple implementation of that would be to form a $3 \times 3$ tower cluster in the centre of the window and compare this to nearest and next-to-nearest neighbours in order to determine whether it is a local maximum.

Another approach is based on Gaussian filtering [6]. Here towers are summed with a weight which decreases as a Gaussian function of the distance from the centre of the cluster. The motivation is that more distant towers are more likely to have a larger (fractional) contribution from pile-up or other jets, so are given a lower weighting.

Figure 11 shows single jet turn-on curves for a simple sliding window algorithm and two Gaussian jets. All are implemented within a $9 \times 9$ window. It can be seen that most of the options have similar turn-ons, with the smaller Gaussian being slightly slower to reach full efficiency and hence requiring a lower threshold.

\section{The Global Feature Extractor Module}

The Global Feature Extractor (gFEX) is currently under study as an optional addition to the L1Calo system. It is intended to identify large-area jets using algorithm window sizes up to $1.8 \times 1.8$ and detector data with a granularity of $0.2 \times 0.2$. The architecture will permit event-by-event local pile-up suppression using $\eta$ dependent baseline subtraction 


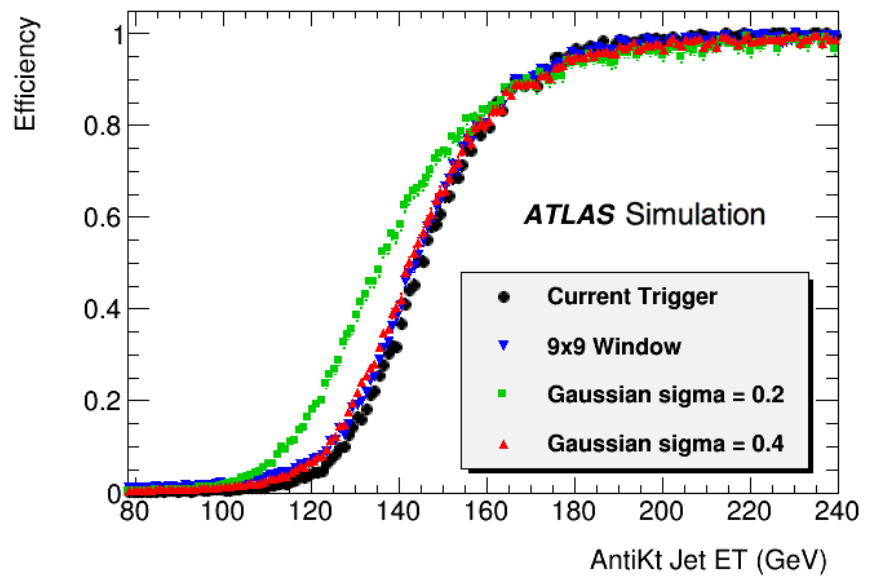

Fig. 11. Single jet turn-on curves for different jet algorithms. In each case an offline anti- $k_{T}$ jet was considered to have been found if there was a trigger jet within $\Delta R<0.4$. The thresholds for the different algorithms were chosen so that the $90 \%$ efficiency points would correspond to that of the current algorithm with a $100 \mathrm{GeV}$ threshold.

techniques. The gFEX architecture is suitable for other algorithms with the same granularity, including $E_{T}^{\text {miss }}$ and $\Sigma E_{T}$.

The gFEX system is implemented in a single ATCA shelf with a dual-star backplane fabric. Two gFEX modules each cover half the calorimeter $\eta$ range. Two Hub modules located in the fabric slots of the shelf perform timing distribution, read-out and control. Input data are carried to the gFEX modules on optical fibres which are routed via rear transition modules to the main boards, where the signals are converted to electrical signals in receiver modules and distributed to processor FPGAs.

A conceptual layout of the gFEX module is shown in Figure 12. Each gFEX module receives data for six $\eta$ rings from both calorimeters with up to 32 fibres per ring, giving a total of up to 192 fibres per module. The input fibres are connected to 16 12-channel parallel-optic receivers, from which the data are distributed to the processor FPGAs.

\section{CONCLUSIONS}

$\mathbf{T}$ HE Phase-I upgrade of the ATLAS Level-1 Calorimeter Trigger will allow the ATLAS experiment to efficiently trigger and record data at instantaneous luminosities that are up to three times that of the original LHC design while maintaining trigger thresholds close to those used in the initial run of the LHC. New Level-1 calorimeter feature extraction processors will be incorporated to allow finer granularity data from the Liquid Argon Calorimeter to be used to enhance trigger selectivity. The techniques described here will improve electron, photon, and tau selection; more sophisticated and larger-area algorithms to be used to improve jet selection; and improved pile-up corrections to be used for missing momentum reconstruction.

\section{REFERENCES}

[1] ATLAS Collaboration, Technical Design Report for the Phase-I Upgrade of the ATLAS TDAQ System, CERN-LHCC-2013-018, ATLAS-TDR-023, 2013.

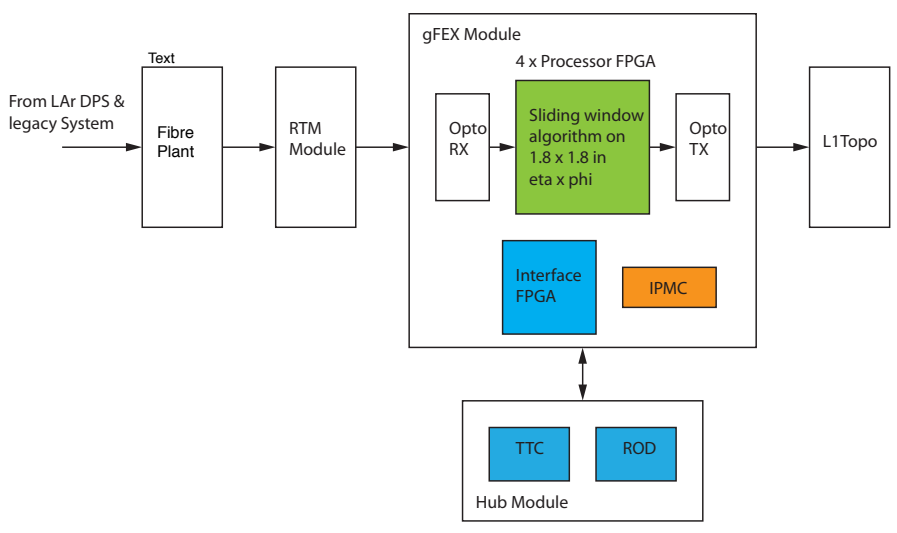

Fig. 12. gFEX conceptual board layout.

[2] R. Achenbach et al., The ATLAS Level-1 Calorimeter Trigger, JINST 3 P03001, 2008.

[3] B. Åsman et al., The ATLAS Level-1 Calorimeter Trigger: PreProcessor implementation and performance, JINST 7 P12008, 2012.

[4] E. F. Eisenhandler, ATLAS Level-1 Calorimeter Trigger Algorithms, ATLDAQ-2004-011, 2004.

[5] ATLAS Collaboration, ATLAS Liquid Argon Calorimeter Phase-I Upgrade Technical Design Report, CERN-LHCC-2013-017, ATLAS-TDR022, 2013.

[6] Y.-S. Lai and B. A. Cole, Jet reconstruction in hadronic collisions by Gaussian filtering, arXiv:0806.1499 [nucl-ex]. 